\title{
Defining end-systolic pressure for single-beat estimation of right ventricle-pulmonary artery coupling: simple... but not really
}

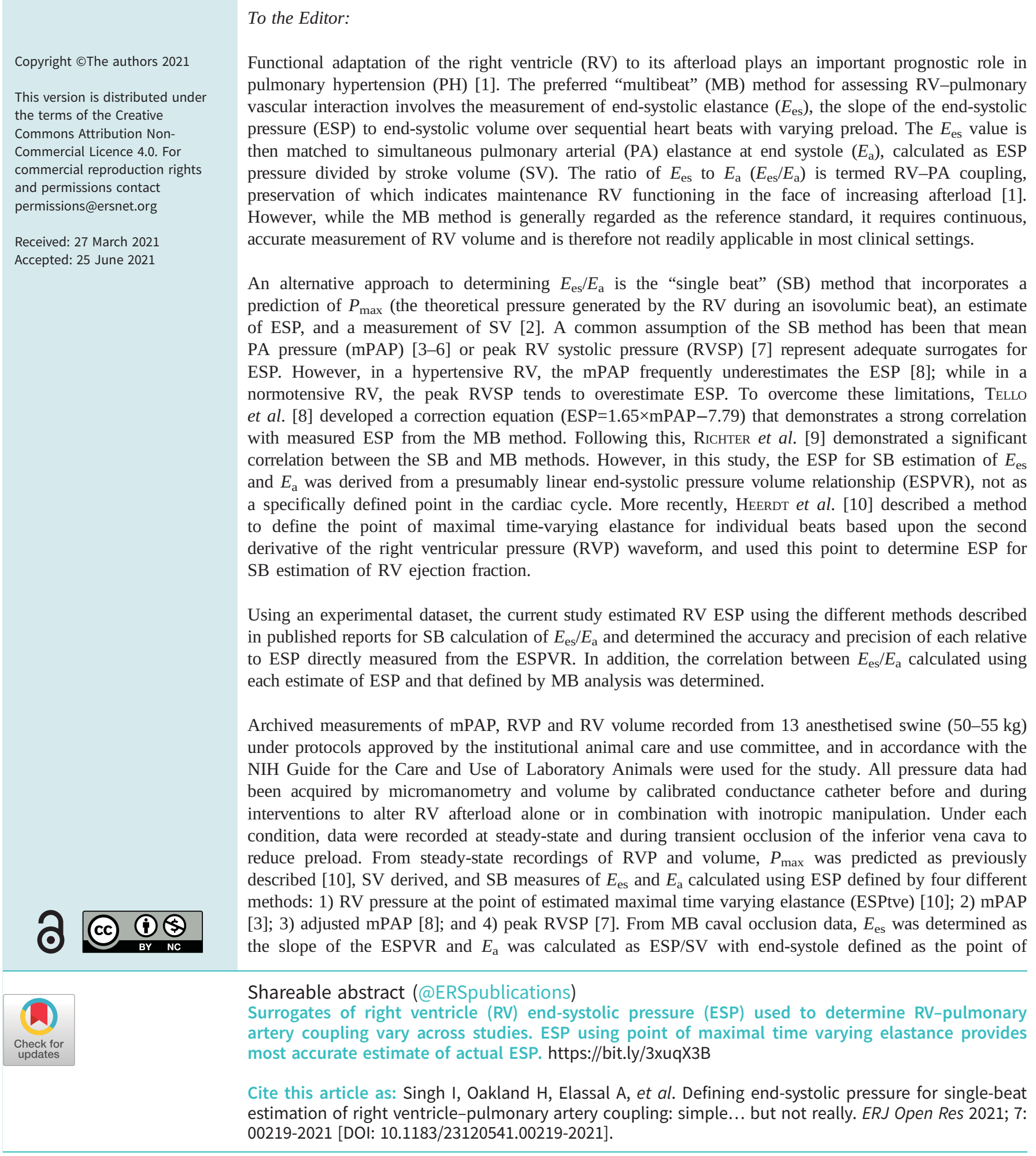



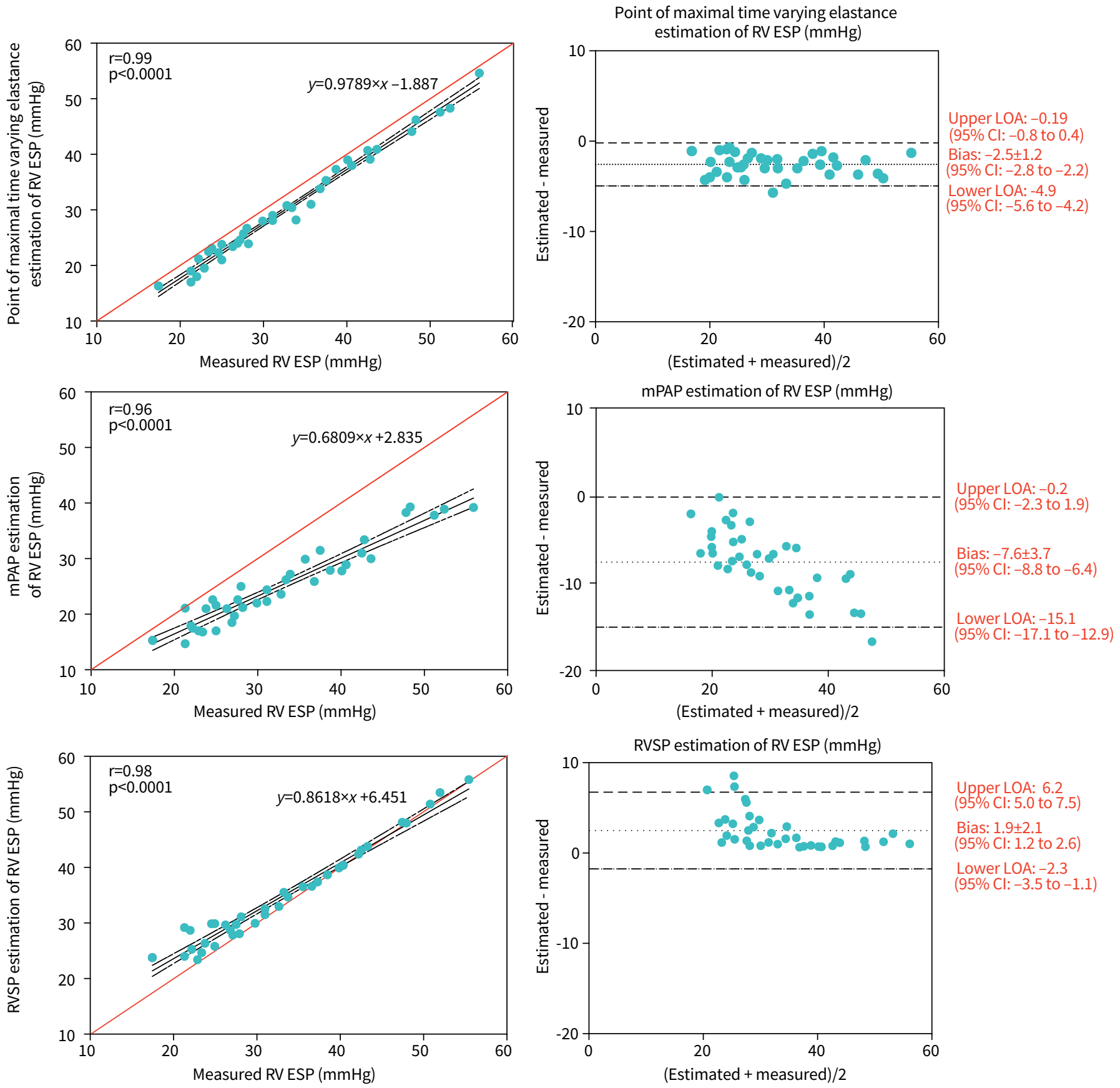

Upper LOA: 6.2 $95 \%$ Cl: 5.0 to 7.5$)$

Bias: $1.9 \pm 2.1$

(95\% Cl: 1.2 to 2.6$)$

Lower LOA: -2.3

$95 \% \mathrm{Cl}:-3.5$ to -1.1

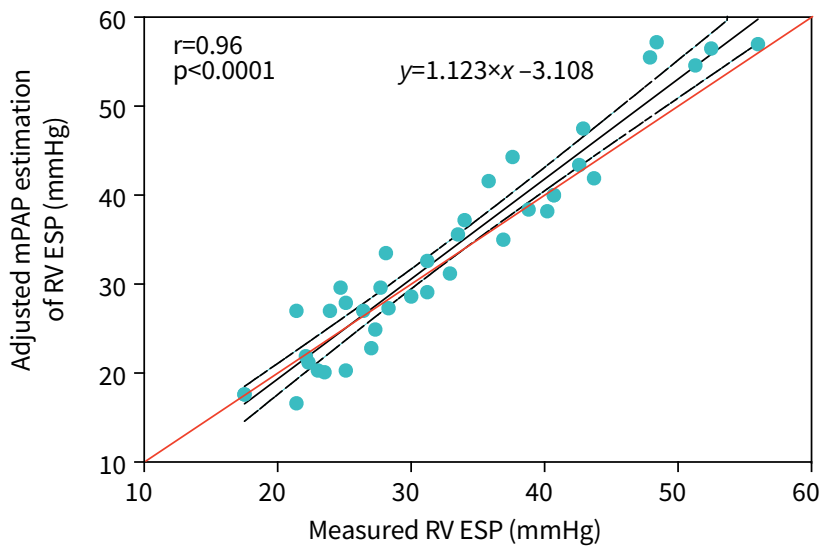

Adjusted mPAP estimation of RV ESP ( $\mathrm{mmHg}$ )

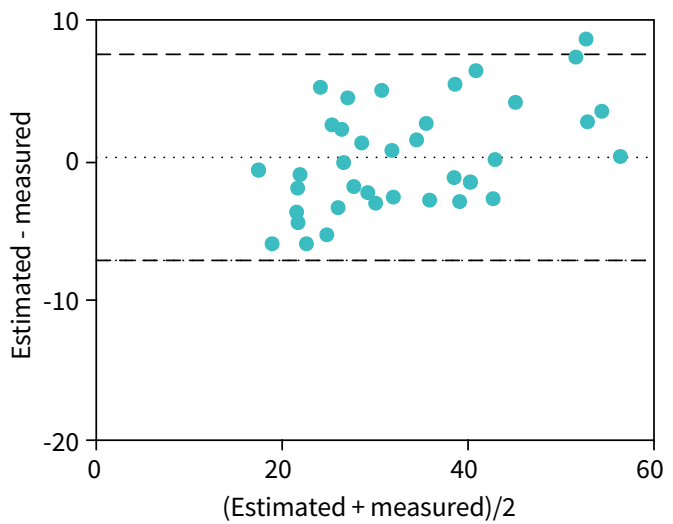

Upper LOA: 7.8 ( $95 \%$ Cl: 5.8 to 9.7 )

Bias: $0.93 \pm 3.5$

(95\% Cl: -6.0 to -4.1 )

Lower LOA: -5.9 (95\% Cl: -7.8 to -3.9$)$ 
FIGURE 1 Correlation between measured right ventricle end-systolic pressure (RV ESP) and estimated RV ESP using different single beat methods. Data presented as means and 95\% confidence intervals of the linear regression functions. Red line denotes the line of identity (left-hand panels). Bland-Altman plot showing the mean difference between methods (bias) and 95\% limits of agreement (LOA) and their respective confidence intervals between estimated and measured RV ESP (right-hand panels). mPAP: mean pulmonary arterial pressure; RVSP: right ventricle systolic pressure.

maximal pressure/volume ratio [11]. A dataset was constructed containing 39 simultaneous measures (three from each animal) of ESP defined by different methods, SB $E_{\mathrm{es}}$ and $E_{\mathrm{a}}$ calculated using the different measures of ESP, and MB $E_{\mathrm{es}}$ and $E_{\mathrm{a}}$.

Comparison between measured RV ESP and that defined by different SB methods was performed using linear regression to define correlation, Bland-Altman plots to define accuracy and precision of estimated data, and four quadrant concordance plots with a $10 \%$ zone of exclusion to assess uniformity of directional change [12]. Measurements were considered potentially interchangeable when the average difference between them (bias) was $<10 \%$ of the mean of measured ESP values, and the overall error calculated as: (bias standard deviation $\times 1.96$ )/mean of all data compared was $\leqslant 30 \%$. These criteria are consistent with previously reported method comparison studies in swine involving haemodynamics [13]. Statistical analyses were performed using GraphPad Prism 9 (GraphPad Software, LLC, La Jolla, CA, USA) and SAS 9.4 (SAS Institute Inc., Cary, NC, USA).

There was strong correlation between measured ESP and all estimates (figure 1). Bland-Altman analysis indicated that the ESPtve demonstrated acceptable accuracy and precision with a bias of $-2.5 \pm 1.2 \mathrm{mmHg}$ ( $8 \%$ of the mean of measured ESP values) and an overall mean error of $8 \%$. Adjusted mPAP provided an ESP estimate that was slightly more accurate (bias $=0.9 \pm 3.5 \mathrm{mmHg}, 3 \%$ of the measured ESP mean) but less precise (mean error=21\%). In contrast, the poorest ESP estimate was mPAP which was neither accurate (bias $=-7.6 \pm 3.8 \mathrm{mmHg}, 21 \%$ of the measured ESP mean) or precise (mean error=26\%). Concordance between measured ESP and the different estimates was 100\% except for RVSP (92\%, data not shown).

With pooled data, the only difference between $\mathrm{MB}$ and $\mathrm{SB} E_{\mathrm{es}} / E_{\mathrm{a}}$ occurred when mPAP was used as a surrogate for ESP (ANOVA on ranks with all comparisons to $\mathrm{MB} E_{\mathrm{es}} / E_{\mathrm{a}}$, data not shown). However, for individual data points significant correlation with $\mathrm{MB} E_{\mathrm{es}} / E_{\mathrm{a}}$ was evident for only the ESPtve (r=0.5, $\mathrm{p}=0.0009)$ and adjusted mPAP $(\mathrm{r}=0.3, \mathrm{p}=0.02)$ methods.

The present study demonstrates that among the different methods for estimating ESP, the approach based upon approximation of the point of maximal time varying elastance (ESPtve) provided greatest accuracy and precision while mPAP provided the worst. However, when adjusted using the equation reported by TELLo et al. [8], mPAP did yield an estimate of ESP that was accurate and acceptably precise (figure 1). In contrast, although peak RVSP demonstrated strong linear correlation with measured ESP when RVP was high, the relationship overall appears nonlinear with RVSP overestimating the measured ESP when RVP was low (figure 1). Presumably, this reflects the fact that with a normotensive RV there is continued ejection well beyond the point of peak RVSP due to the high capacitance and low resistance pulmonary circulation [14], resulting in ESP being reached later in the cardiac cycle. Overall, the study results highlight previous reports [8] that although mPAP is correlated with RV ESP and has been frequently used as a surrogate [3-6], it progressively underestimates ESP as RVP rises (figure 1).

Across all methods, a significant relationship between both pooled and individual SB and MB $E_{\mathrm{es}} / E_{\mathrm{a}}$ data was evident only for ESPtve and adjusted mPAP. This underscores the importance of considering how ESP was defined when interpreting absolute values for $E_{\mathrm{es}} / E_{\mathrm{a}}$ reported in the literature.

Results from the current study need to be interpreted in the context of limitations. Data for analysis were compiled from 13 anesthetised, ventilated pigs with acute pulmonary vasoconstriction. Further work is required for clinical validation of the study results across different $\mathrm{PH}$ phenotypes. In addition, while the study results are broadly consistent with the concept that $\mathrm{SB}$ measures of $E_{\mathrm{es}} / E_{\mathrm{a}}$ using ESPtve can effectively represent the MB reference standard, specific definition of the accuracy and precision of this representation is beyond the scope of the current investigation.

In summary, the current study demonstrates that estimation of ESP using an RVP-based approximation of the point of maximal time varying elastance can provide an accurate and precise value. This method eliminates the need for simultaneous measurement of mPAP when performing SB analysis of RV $E_{\mathrm{es}} / E_{\mathrm{a}}$. 
Inderjit Singh ${ }^{1}$, Hannah Oakland $\oplus^{1}$, Ahmed Elassal ${ }^{2}$ and Paul M. Heerdt $\circledast^{2}$

${ }^{1}$ Division of Pulmonary, Critical Care, and Sleep Medicine, Dept of Medicine, Yale New Haven Hospital and Yale School of Medicine, New Haven, CT, USA. ${ }^{2}$ Dept of Anaesthesiology, Division of Applied Hemodynamics, Yale New Haven Hospital and Yale School of Medicine, New Haven, CT, USA.

Corresponding author: Inderjit Singh (inderjit.singh@yale.edu)

Submitted article, peer reviewed

Author contributions: I. Singh, H. Oakland and P.M. Heerdt contributed to conception and design of the work, interpretation of the data and writing. A. Elassal and P.M. Heerdt contributed to data acquisition and analysis. All authors approved the final version of the manuscript and are accountable for all aspects of the work.

Conflict of interest: I. Singh has nothing to disclose. H. Oakland has nothing to disclose. A. Elassal has nothing to disclose. P.M. Heerdt reports grants from NTT Research and IMPERATiV, and an ownership interest in RVMetrics, LLC (dissolved in 2020), outside the submitted work.

Support statement: This study was support by National Institutes of Health grant 2T32GM086287-11 (to A. Elassal). Funding information for this article has been deposited with the Crossref Funder Registry.

\section{References}

1 Vonk-Noordegraaf A, Haddad F, Chin KM, et al. Right heart adaptation to pulmonary arterial hypertension: physiology and pathobiology. J Am Coll Cardiol 2013; 62: 25 Suppl., D22-D33.

2 Brimioulle $\mathrm{S}$, Wauthy $\mathrm{P}$, Ewalenko $\mathrm{P}$, et al. Single-beat estimation of right ventricular end-systolic pressure-volume relationship. Am J Physiol Heart Circ Physiol 2003; 284: H1625-H1630.

3 Philip JL, Chesler NC. Know your limitations: assumptions in the single-beat method for estimating right ventricular-pulmonary vascular coupling. Am J Respir Crit Care Med 2018; 198: 707-709.

4 Rajaratnam A, Rehman S, Sharma $\mathrm{P}$, et al. Right ventricular load and contractility in HIV-associated pulmonary hypertension. PLOS ONE 2021; 16: e0243274.

5 Contijoch F, Wong D, Igata S, et al. Association between preoperative dynamic measures of vascular load and postoperative hemodynamics in patients with chronic thromboembolic pulmonary hypertension after pulmonary thromboendarterectomy. Ann Am Thorac Soc 2021; 18: 551.

6 Singh I, Rahaghi FN, Naeije R, et al. Right ventricular-arterial uncoupling during exercise in heart failure with preserved ejection fraction: role of pulmonary vascular dysfunction. Chest 2019; 156: 933-943.

7 Metkus TS, Mullin CJ, Grandin EW, et al. Heart rate dependence of the pulmonary resistance x compliance (RC) time and impact on right ventricular load. PLOS ONE 2016; 11: e0166463.

8 Tello K, Richter MJ, Axmann J, et al. More on single-beat estimation of right ventriculo-arterial coupling in pulmonary arterial hypertension. Am J Respir Crit Care Med 2018; 198: 816-818.

9 Richter MJ, Peters D, Ghofrani HA, et al. Evaluation and prognostic relevance of right ventricular-arterial coupling in pulmonary hypertension. Am J Respir Crit Care Med 2020; 201: 116-119.

10 Heerdt PM, Kheyfets V, Charania S, et al. A pressure-based single beat method for estimation of right ventricular ejection fraction: proof of concept. Eur Respir J 2020; 55: 1901635.

11 Sagawa K ML, Suga H, Sunagawa K. Cardiac contraction and the pressure-volume relationship. New York, Oxford University Press, 1988.

12 Thiele RH, McMurry TL. Data agnosticism and implications on method comparison studies. Anesth Analg 2015; 121: 264-266.

13 Berlin DA, Manoach S, Oromendia C, et al. Automated expiratory ventilation assistance through a small endotracheal tube can improve venous return and cardiac output. Intensive Care Med Exp. 2019; 7: 6.

14 Richter MJ, Hsu S, Yogeswaran A, et al. Right ventricular pressure-volume loop shape and systolic pressure change in pulmonary hypertension. Am J Physiol Lung Cell Mol Physiol 2021; 320: L715-L725. 